\title{
Estudo bibliométrico: Novas tecnologias em marketing - período 2010 -2018
}

\author{
Bibliometric study: New technologies in marketing - from 2010 to 2018 \\ Estudio bibliométrico: Nuevas tecnologías en marketing - período 2010-2018
}

\section{Resumo}

Este estudo tem como objetivo avaliar a produção científica sobre a adoção de novas tecnologias para auxiliar o marketing na promoção de estratégias que facilitem as organizações brasileiras a se tonarem mais competitivas no mercado. A metodologia possui uma abordagem quantitativa visando quantificar os modelos utilizados, através das publicações em eventos (EnANPAD, EMA e CLAV) no período de 2010 a 2018. O estudo teve como resultado a restrição da adoção de novas tecnologias a determinados tipos de produtos ou serviços, bem como de uma revolução rápida desta tecnologia no mercado. Observou-se que os modelos mais utilizados na análise foram o TAM e UTAUT2, o que demostra a importância da utilização de modelos para avaliar o consumo e a aceitação dos consumidores aos produtos ofertados. Os resultados obtidos restringem-se a 37 artigos apresentados em eventos como EMA e EnANPAD e a CLAV no período de 2010 a 2018 na qual o tema foi estudado. O tema é contemporâneo no Brasil, e apresenta limitações que prejudicam um estudo mais robusto, porém é emergente e existe um grande potencial para o desenvolvimento de estudos empíricos que tratem da adoção de novas tecnologias e Marketing.

Palavras-chave: Adoção; Novas tecnologias; Marketing; Modelos.

\begin{abstract}
The main goal of this study is to evaluate the scientific production on the adoption of new technologies to help the marketing field on the promotion of strategies that aim to help brazilian organizations to become more competitive. A quantitative methodology was employed to measure the models used, throughout publications (EnANP AD, EMA and CLA V) from 2010 to2018. The results found were the restriction on the adoption of new technologies to some types of products or services, and the fast evolution of said technologies in the market. It is possible to see that the most utilized models in the analysis were T AM and UT AUT2, which shows the importance of these model's usage to evaluate the consumption and acceptance from consumers to products offered to them. Only 37 articles were presented in events such as EMA, EnANP AD and CLA V from 2010 to 2018. This subject is contemporary in Brazil, and has limitations that jeopardize deeper studies, but it is emerging and it has a lot of potential for greater studies that approach the theme of new technologies in marketing.
\end{abstract}

Keywords: Adoption; New technologies; Marketing; Models.

\section{Resumen}

Este estudio tiene como objetivo evaluar la producción científica sobre la adopción de nuevas tecnologías para auxiliar el marketing a promover estrategias que posibiliten a las organizaciones brasileñas ser más competitivas en el mercado. La metodología aplica un enfoque cuantitativo buscando hacer un recuento de los modelos utilizados, por medio de publicaciones en eventos (EnANPAD, EMA y CLAV) en el período de 2010 a 2018. El estudio constató la restricción de la adopción de nuevas tecnologías a ciertos tipos de productos o servicios, así como una rápida revolución de esta tecnología en el mercado. Se observó que los modelos más utilizados en el análisis fueron el TAM y el UTAUT2, lo que demuestra la importancia de que se utilicen modelos para evaluar el consumo y la aceptación de los consumidores a los productos ofertados. Los resultados obtenidos se limitan a 37 artículos presentados en eventos como EMA y EnANPAD, y el CLAV, en el período de 2010 a 2018 en el que el tópico fue estudiado. El tema es contemporáneo en 
Brasil, presentando limitaciones que dificultan un estudio más robusto, pero está emergiendo y existe un gran potencial para el desarrollo de estudios empíricos que aborden la adopción de nuevas tecnologías y Marketing.

Palabras clave: Adopción; Nuevas tecnologías; Marketing; Modelos.

\section{Introdução}

As novas tecnologias atualmente emergem das rápidas mudanças, advindas da globalização. A rapidez em que caminham as inovações faz com que surjam novas organizações de sucesso, tornando obsoletas e ultrapassadas as que não conseguem evoluir e adaptar-se ao novo ambiente (Ferraz, et al., 1995). A nova era de informação e tecnologia traz para as organizações uma necessidade constante de adequar estratégias que contemplem serviços e produtos, de maneira que possam ser competitivas em face à abertura dos mercados. Entretanto, surge uma série de estudos sobre as novas tecnologias e o Marketing no campo das organizações e de como ocorrem as evoluções em tempos em que as tecnologias e as comunicações precisam caminhar juntas para uma melhor eficiência produtiva.

A inserção de novas tecnologias nas organizações é um processo que vem cada vez mais ocorrendo, de forma a possibilitar a modernização, melhorar o atendimento aos clientes e permitir que seja criado um sistema de informações de controle e gerenciamento de forma a gerar menores custos. Tudo isso devido ao atual cenário de intensa concorrência que o mercado vem apresentando.

O problema estudado foca na exploração dos aspectos da adoção de novas tecnologias e do Marketing em função de que as sociedades de consumidores/clientes estão mais exigentes no que tange à otimização do tempo e buscam soluções rápidas para o dia-a-dia.

Segundo Souza e Luce 2003, uma preocupação das organizações é de como os consumidores recebem as novas tecnologias por elas adotadas, uma vez que as mesmas visam à satisfação de seus clientes, sendo que alguns indivíduos podem ter uma boa impressão acerca da tecnologia que está sendo adotada, como também pode ocorrer de haver insegurança ou desconforto diante da novidade apresentada.

De acordo com os artigos analisados na realização do presente estudo, se destacaram alguns modelos e teorias que basearam a adoção de novas tecnologias para auxiliar o Marketing nas organizações no Brasil. Dentre essas teorias/modelos pode-se salientar: Theory of Reasoned Action (TRA) e a Theory of Planed Behavior (TPB); Modelo de Aceitação Tecnológica (Technology Acceptance Model - TAM); Unified Theory of Acceptance and Use of Technology 2 - UTAUT2; A Mobile Crowd Sensing (MCS); Teoria da Ação Racionalizada-Fishbein e Ajzen (1975); Teoria da Difusão da Inovação (DOI) -Rogers (1983, 1985); Teoria Cognitiva Social-Bandura (1986); Teoria do Comportamento Planejado (TPB) -Ajzen (1991); etc.

Esses modelos e teorias servem como suporte ao Marketing na adoção de novas tecnologias, são eles que embasam e estruturam as inovações trazidas pelas organizações, de forma que tudo o que for criado e implantado em uma organização deve ser explicado através de uma ou mais teorias e estruturado através de modelos previamente existentes.

Dito isto, a produção científica apresenta a adoção de novas tecnologias e o Marketing como algo importante no cenário brasileiro, apesar de haver um número reduzido de pesquisas apresentadas em eventos que contemplam este tema, o que demostra uma lacuna no que tange a modernização das organizações. A análise deste tema de estudo parte de artigos apresentados em eventos dos Encontros da Associação Nacional de Pós-Graduação e Pesquisa em Administração (EnANPAD), Encontro de Marketing (EMA) e Congresso Latino-Americano de Varejo (CLAV) no período entre 2010 a 2018.

Nesse sentido, cabe às organizações atender às demandas dos consumidores inserindo no mercado produtos e serviços que sejam eficientes e capazes de suprir a necessidade de seus clientes. 
O Objetivo deste estudo está em avaliar a produção científica em torno da adoção de novas tecnologias e os principais modelos utilizados para auxiliar o Marketing na promoção de estratégias que auxiliam as organizações brasileiras a se tonarem mais competitivas no mercado.

\subsection{A adoção de novas tecnologias e os principais modelos utilizados para explicar este fenômeno}

Segundo Gonçalves (1993), a implantação de novas tecnologias nas empresas vem se intensificando há décadas, no sentido de melhor atender ao cliente bem como melhorar o controle e gerenciamento, objetivando a redução de custos.

Quanto ao conceito de tecnologia, Gonçalves (1993), define que a mesma consiste em um conjunto integrado de conhecimentos, técnicas, ferramentas e procedimentos de trabalho que podem até mesmo serem manuais, aplicados na produção econômica de bens e serviços. Assim, a tecnologia é considerada como um produto da ciência que envolve um conjunto de instrumentos, métodos e técnicas que além de resolver problemas trazem eficiência, praticidade e redução de custos para as organizações.

Assim, de acordo com Gonçalves (1993), a tecnologia deve ser vista muito além dos equipamentos, máquinas e computadores, mas sim deve ser vista a partir de um sistema técnico, que engloba as ferramentas, as técnicas e os métodos utilizados pelas organizações para realizar suas tarefas. Destaca que também existe um sistema social, que envolve as necessidades, expectativas e sentimentos sobre o trabalho, de forma que esses dois sistemas devem ser simultaneamente otimizados, tornando a tecnologia uma representação de um conjunto de características específicas do sistema técnico.

Conforme Masiero (2007), as novas tecnologias devem ser percebidas como um processo de modernização no que se refere à inserção de produtos que possam otimizar e melhorar o acesso do consumidor com maior rapidez e eficiência. Para tanto, as organizações precisam inovar o que compreende a introdução de um novo modelo tecnológico que permita novas práticas organizacionais. Ainda segundo Masiero (2007) as formas de inovar compreendem na introdução de um novo modelo de produção, na inovação de um produto, no surgimento de novas oportunidades no mercado, na utilização de novas fontes de matérias-primas, de fatores de produção e de produtos semi-industrializados, e também em novos tipos de organização e gestão.

Para além dos conceitos existentes de tecnologia, o que se preocupa nesse estudo é entender como os consumidores interagem e aceitam as mudanças advindas da adoção de novas tecnologias pelas organizações para auxiliar o Marketing na prestação dos serviços.

Nesse aspecto, de acordo com Wohnrath (2007), é importante levar em consideração como os indivíduos (clientes/consumidores), recepcionam a implantação das novas tecnologias nas organizações, destacando a importância da preparação e do envolvimento do fator humano no processo de desenvolvimento e implantação das novas tecnologias, de forma a haver uma integração sistêmica entre os recursos tecnológicos e humanos.

Nesse sentido também se manifesta Araújo 2019 apud Bagozzi e Lee (1999) e Winner (1994) afirmando o "choque" sentimental que ocorre com os consumidores ao receberem as tecnologias, faz com que os mesmos se manifestem positivamente ou adotem uma postura negativa, de forma que se percebe a existência de fatores motivadores e inibidores quanto à adoção de novas tecnologias.

Diante disso, o presente artigo pretende demonstrar que para uma empresa conseguir sobreviver no cenário atual, ela deve ter diferenciais que a destaquem das demais de seu ramo, pois não basta se manter no mercado, é preciso se sobressair e se desenvolver tecnologicamente, isto significa ter capacidade de liderança no ambiente em que está inserida, por isso a adoção de novas tecnologias se faz necessária e indispensável.

Porter (1989) destaca que por mais que uma empresa tenha inúmeros pontos fortes e fracos em relação a seus concorrentes, as empresas bem-sucedidas sempre obedecem a padrões bem definidos de comportamento. O principal foco está 
na diferenciação, na utilização do Marketing como um aliado na adoção de novas tecnologias, na criação de produtos e serviços com menores custos e eficientes.

Dessa forma, a relação entre a adoção de novas tecnologias e o Marketing é importante principalmente por contribuir para a diferenciação dos negócios da empresa, situação que propicia a otimização da produtividade, melhoria da comunicação, aumento da agilidade, melhora na qualidade dos produtos e dos métodos de controle e planejamento, permitindo que a empresa desempenhe atividades estrategicamente importantes com maior eficiência e melhor que a concorrência.

Entretanto, de acordo com Araújo 2019 apud Ferreira, Rocha \& Silva, 2014; Peres, Muller \& Mahajan, 2010, existem poucos estudos na literatura atual que tratem da adoção de novas tecnologias, o que leva diversos autores a clamarem por novas pesquisas sobre $\mathrm{o}$ assunto.

Assim, o presente artigo tem como principal objetivo analisar em eventos do EnAMPAD, EMA e CLAV, trabalhos que tratem da adoção de novas tecnologias pelas empresas para auxiliar o Marketing no Brasil, de forma a trazer esse tema ao cenário atual através da presente análise bibliométrica, expandindo um pouco mais a literatura quanto a esse tema.

No presente estudo também foram analisadas diferentes teorias e modelos que inseriram a tecnologia a serviço do Marketing, como por exemplo, as teorias advindas das áreas de sistemas de informação e de marketing, as quais propõe um modelo de decisão de compra de TI por organizações, quais sejam: Theory of Reasoned Action (TRA) e a Theory of Planed Behavior (TPB) e de maneira complementar o Modelo de Aceitação Tecnológica (Technology Acceptance Model - TAM) de Davis (1989), o qual tem sido utilizado para avaliar a relação de aceitação entre consumidores e produtos tecnológicos.

Ao analisar as teorias e modelos utilizados nos artigos estudados, é possível observar que os autores procuraram desenvolver modelos comportamentais os relacionando com a intensão de uso do aplicativo.

Outra teoria muito utilizada nos artigos analisados é a Unified Theory of Acceptance and Use of Technology 2 UTAUT2, que possibilita um entendimento dos direcionadores de aceitação tecnológica, da autoria de Venkatesh et. al (2003). Conforme aduz Mourad et al 2018, trata-se de um modelo teórico projetado para explicar e prever a adoção da tecnologia em contexto de consumo, além de ser um modelo global e integrativo, por ser incorporado a ele as principais variáveis de modelos teóricos anteriores sobre aceitação de tecnologia, provando ser um modelo de sucesso para estudar a aceitação e uso de tecnologia em diversos contextos.

A Mobile Crowd Sensing (MCS), é um outro modelo encontrado em Guo et al. (2015) e consiste num novo paradigma sensorial que capacita os cidadãos comuns para contribuir com dados detectados ou gerados a partir de seus dispositivos móveis.

Além disso, outras teorias e modelos foram observados nos artigos analisados, tais como Teoria da Ação RacionalizadaFishbein e Ajzen (1975); Teoria da Difusão da Inovação (DOI) -Rogers (1983, 1985); Teoria Cognitiva Social-Bandura (1986); Teoria do Comportamento Planejado (TPB) -Ajzen (1991); entre outros tantos modelos e teorias utilizados.

Portanto, percebe-se que a adoção de novas tecnologias tanto pode ser aceita com tranquilidade pelos consumidores/clientes de determinada organização, como também pode acontecer de não haver essa aceitação tão instantânea, seja por medo, insegurança ou dúvida de que a inserção da nova tecnologia realmente seja eficiente e segura nas transações e nos negócios. Nesse sentido, se destaca a importância do presente estudo, ao analisar bibliometricamente diversos artigos que tratam do tema, destacando os modelos e teorias mais utilizados na adoção das novas tecnologias pelas organizações. Com isso, pode-se aprofundar o conhecimento a respeito desse assunto tão importante e indispensável nos últimos tempos, demonstrando que a adoção de novas tecnologias realmente é importante e que cada vez mais está sendo realizada, uma vez que o mercado exige diante da rápida evolução dos sistemas, o que vem a provocar a necessidade das organizações a se reestruturarem estrategicamente, aumentando assim sua produtividade, reduzindo custos e se mantendo ativas no cenário atual. 


\section{Metodologia}

O presente estudo foi desenvolvido a partir de uma pesquisa bibliométrica, que para Macedo et al (1999) auxilia a verificar o estágio que uma pesquisa em determinada área se encontra. Esta forma de estudo vem auxiliar o pesquisador na qualificação do tema abordado, ou seja, traz suporte no que tange a adoção das novas tecnologias no Marketing apresentando o que está sendo discutido na academia.

A abordagem metodológica é quantitativa, visto que procurou quantificar algumas variáveis referentes à produção científica sobre a adoção de novas tecnologias em Marketing, tendo como principal objetivo ampliar o conhecimento a partir das publicações em eventos (EnANPAD, EMA e CLAV) no período de 2010 a 2018. A coleta de artigos foi realizada através da busca pelo termo "Novas Tecnologias" "Marketing" nos títulos, resumos e palavras-chave, nas bases de dados dos eventos selecionados, seguida da leitura dos resumos de cada artigo selecionado, a fim de verificar a ênfase em questões que se vinculam ao tema das novas tecnologias no marketing. A seleção finalizou em um conjunto de 37 artigos apresentados no total. Buscaram-se trabalhos apenas apresentados e publicados em anais dos eventos supracitados, sendo analisados 35 artigos na língua portuguesa e 02 artigos na língua inglesa. A coleta de dados foi realizada num período de dois meses e seguida pela tabulação, através da ferramenta Software Excel 2018. Todos os dados tabulados foram conferidos e analisados pelos pesquisadores para enfim realizar as considerações finais sobre o tema abordado.

Portanto, este estudo realizou uma avaliação bibliométrica, analisou e verificou artigos entre 2010 a 2018 em alguns eventos brasileiros na área de Marketing. Este artigo está estruturado da seguinte maneira: (1) Introdução (contextualização teórica da adoção de novas tecnologias e os principais modelos que são utilizados para explicar este fenômeno); (2) Metodologia; (3) Resultados referentes aos eventos sobre o tema em estudo; (4) Discussão (os autores que mais publicaram nos periódicos estudados EnANPAD, EMA e CLAV); (5) considerações finais.

\section{Resultados e Discussão}

A análise de dados deste estudo foi realizada a partir de artigos apresentados e publicados em anais dos Encontros da Associação Nacional de Pós-Graduação e Pesquisa em Administração (EnANPAD), Encontro de Marketing (EMA) e Congresso Latino-Americano de Varejo (CLAV) no período entre 2010 a 2018. Foram mapeados cerca de 37 artigos apresentados nos referidos eventos com o tema Novas Tecnologias e Marketing. Esta análise está baseada em estudos de Vieira e Gava (2006), Gomes et al. $(2009 ; 2011)$ uma vez que cada tabela a seguir apresenta dados que (1) avalia a produção e apresentação anual de artigos.

Assim, foi possível verificar a importância e a evolução das novas tecnologias como auxilio ao Marketing e qual foi a contribuição para as organizações, além do tipo de artigo apresentado pelos pesquisadores nestes eventos (empíricos e teóricos); (2) verificar a repetição de autores em demais artigos produzidos pertinente ao tema nos eventos selecionados durante o período de 2010 -2018; (3) analisar os autores teóricos que mais apareceram nos artigos referentes ao temas supracitados; (4) analisar os modelos mais utilizados de acordo com os eventos analisados; (5) analisar os produtos os quais foram utilizados com as novas tecnologias auxiliando o Marketing.

\subsection{Produção anual de artigos apresentados em eventos}

De acordo com a Tabela 1, dentre os três eventos (EnANPAD, EMA e CLAV) analisados no estudo, somam 37 artigos apresentados entre os anos 2010 e 2018, sobre adoção de novas tecnologias e o Marketing. De acordo com a tabela fica evidenciado que este tema não foi abordado em muitos eventos do EMA, mesmo que este encontro trate especificamente sobre a importância e influência do Marketing nas organizações. Neste evento apenas $04(11,2 \%)$ artigos foram apresentados no 
decorrer dos anos em estudo, chamando a atenção de que em 2010 (02 artigos) e 2018 (02 artigos), apresentando, portanto, um período em que não houveram estudos referentes a este item.

No evento EnANPAD foram apresentados 06 (16,6\%) artigos no decorrer dos anos em estudo, sendo que nos anos 2010 (01 artigo), 2013 (01 artigo), 2017 (01 artigo) e por fim, 2018 com (02 artigos). Na EnANPAD também não teve muito êxito no que tange ao tema, não demostrando crescimento no decorrer dos anos em estudo.

No caso da CLAV foi o evento que mais teve apresentações no que se refere a adoção de Novas tecnologia no Marketing, foram 26 (72,2\%) artigos dos quais estão distribuídos entre os anos a 2016 a 2018, sendo que não se tem dados referentes aos anos de 2010 a 2015 pois não consta no sistema de informação da CLAV. Na CLAV em 2016 foram apresentados e publicados em anais no ano de 2016 um número de 05 artigos (19,2\%), em 201712 artigos (44,4\%) e $201810 \operatorname{artigos~(36,4\% ).~}$

É possível analisar, portanto, que o Evento CLAV, mesmo não tendo informações em determinado período apresenta o maior índice de estudo sobre o referido tema de acordo com os eventos analisados, demonstrado o maio pico de estudos no ano de 2017 e uma queda no ano 2018. Portanto a soma dos dois eventos que mais trazem estudos sobre o tema abordado são EnANPAD e CLAV representam $32(86,4 \%)$ artigos no total.

Tabela 1 - Publicações sobre adoção de novas tecnologias e marketing.

\begin{tabular}{l|c|c|c|c|c|c|c|c|c|c}
\hline Periódicos & $\mathbf{2 0 1 0}$ & $\mathbf{2 0 1 1}$ & $\mathbf{2 0 1 2}$ & $\mathbf{2 0 1 3}$ & $\mathbf{2 0 1 4}$ & $\mathbf{2 0 1 5}$ & $\mathbf{2 0 1 6}$ & $\mathbf{2 0 1 7}$ & $\mathbf{2 0 1 8}$ & Total \\
\hline EMA & 2 & 0 & 0 & 0 & 0 & 0 & 0 & 0 & 2 & $\mathbf{4}$ \\
\hline ENAMPAD & 2 & 0 & 0 & 1 & 0 & 0 & 0 & 1 & 2 & $\mathbf{6}$ \\
\hline CLAV & 0 & 0 & 0 & 0 & 0 & 0 & 5 & 12 & 10 & $\mathbf{2 7}$ \\
\hline Total & $\mathbf{4}$ & $\mathbf{0}$ & $\mathbf{0}$ & $\mathbf{1}$ & $\mathbf{0}$ & $\mathbf{0}$ & $\mathbf{5}$ & $\mathbf{1 3}$ & $\mathbf{1 4}$ & $\mathbf{3 7}$ \\
\hline
\end{tabular}

Fonte: Autores.

Em relação aos tipos de artigos apresentados nos eventos, observou-se que $54 \%$ dos estudos têm predominância empírica no que tange a utilização de novas tecnologias e a inserção dos mesmos na prestação de serviços e produtos com auxílio do Marketing (Tabela 2). Os artigos em estudo foram apresentados e publicados em anais dos eventos supracitados, tendo uma ressalva no que se refere à CLAV no período de 2010 a 2015 que não tem acesso a artigos apresentados naquele período. Os artigos teóricos sobre o tema iniciaram a partir do ano de 2016 demonstrando, portanto, neste período, um aumento na produção de artigos com intuito de demonstrar as teorias acerca das novas tecnologias e do Marketing. De maneira geral, percebe-se também que os artigos empíricos se mantiveram em leve crescimento até o ano 2018, tendo a ressalva de que alguns períodos não se têm dados. Pode se dizer ainda que até 2018 os artigos empíricos superam os artigos teóricos.

Tabela 2 - Tipos de artigo.

\begin{tabular}{l|c|c|c|c|c|c|c|c|c|c}
\hline Tipo de Artigo & $\mathbf{2 0 1 0}$ & $\mathbf{2 0 1 1}$ & $\mathbf{2 0 1 2}$ & $\mathbf{2 0 1 3}$ & $\mathbf{2 0 1 4}$ & $\mathbf{2 0 1 5}$ & $\mathbf{2 0 1 6}$ & $\mathbf{2 0 1 7}$ & $\mathbf{2 0 1 8}$ & Total \\
\hline Empírico & 04 & 0 & 0 & 01 & 0 & 0 & 02 & 06 & 07 & $\mathbf{2 0}$ \\
\hline Teórico & 0 & 0 & 0 & 0 & 0 & 0 & 03 & 07 & 07 & $\mathbf{1 7}$ \\
\hline Total & $\mathbf{4}$ & $\mathbf{0}$ & $\mathbf{0}$ & $\mathbf{1}$ & $\mathbf{0}$ & $\mathbf{0}$ & $\mathbf{5}$ & $\mathbf{1 3}$ & $\mathbf{1 4}$ & $\mathbf{3 7}$ \\
\hline
\end{tabular}

Fonte: Autores. 


\subsection{Autores que publicaram}

$\mathrm{Na}$ Tabela 3,14 artigos (37,8\%) foram produzidos por mais de dois autores. Isto não parece algo novo para análise bibliométrica, dado que muitos artigos de outros eventos e periódicos este fato é corriqueiro. De acordo com Vieira (1998) também já havia evidenciado um alto nível de publicações de artigos por apenas 02 autores, isto demonstra que não houve mudança significativa neste quesito, ou seja, este autor evidencia esta situação devido ao fato de que a pesquisa geralmente é guiada pelo orientador e orientando, sendo que os artigos que mais se salientaram nesta questão são artigos apresentados e publicados em anais da CLAV entre 2016 e 2018. Estes artigos são provenientes de discentes de graduação e pós-graduação de diferentes instituições de ensino. Também pode ser observado 08 artigos com 03 autores (21\%) e 08 artigos com 04 autores (21\%) totalizando $42 \%$ do total.

Tabela 3 - Quantidade de autores por artigo.

\begin{tabular}{c|c}
\hline Autores & Artigos \\
\hline 01 & 2 \\
\hline 02 & 14 \\
\hline 03 & 8 \\
\hline 04 & 8 \\
\hline 05 & 2 \\
\hline 06 ou mais & 2 \\
\hline
\end{tabular}

Fonte: Autores.

Os autores que mais publicaram sobre a adoção de novas tecnologias e o Marketing entre 2010 e 2018 foram Emílo José Montero Arruda Filho, Rafael Tezza, Wilnei Aldair Scheneider e Carlos Roberto De Rolt e todos eles nos eventos especificos da CLAV 2016 e 2017. Não se tem informação qual instituição, pois não consta no artigo. Os artigos dos referidos autores trazem em seu contexto questões referentes à aplicabilidade da adoção de novas tecnologias, demosntrando nos seus estudos basicamente o comportamento na aceitação dos produtos a serem sugeridos e ainda a dificuldade dos sujeitos no que tange o ajustar de situações frente às mudancas tecnológicas, além, é claro, da motivação na utilização desta nova tecnologia.

Tabela 4 - Autores que mais publicaram na área.

\begin{tabular}{l|c}
\hline Autores & Publicações \\
\hline Emilio José Montero Arruda Filho & 04 \\
\hline Rafael Tezza & 02 \\
\hline Wilnei Aldir Schneider & 02 \\
\hline Carlos Roberto De Rolt & 02 \\
\hline Outros & 103 \\
\hline
\end{tabular}

Fonte: Autores.

Quanto aos modelos mais utilizados observa -se na Tabela 5, a Technology Acceptance Model - TAM e Unified Theory of Acceptance and Use of Technology 2 - UTAUT2 são as que mais aparecem nas descrições dos referidos artigos. Sendo que o 
primeiro (TAM), se trata de uma teoria que trabalha a forma como os usuários aceitam e usam uma tecnologia; é um modelo definido por Davis no sentido de perceber o grau que uma pessoa que utilizar um determinado sistema aumentaria seu desempenho no trabalho. Esse modelo representou 16,20\% de utilização nos artigos analisados. Já o modelo Unified Theory of Acceptance and Use of Technology 2 - UTAUT2, formulado por Venkatech e outros, significa Teoria Unificada de Aceitação e Uso de Tecnologia. O UTAUT tem como objetivo explicar as intenções do usuário de usar um sistema de informações e o comportamento de uso subsequente. A teoria sustenta que existem quatro construtos principais: 1) expectativa de desempenho, 2) expectativa de esforço, 3) influência social e 4) condições facilitadoras. Este modelo representa 8,10\% de utilização nos artigos estudados para desenvolver o presente trabalho.

Tabela 5- Modelos utilizados nos artigos.

\begin{tabular}{l|c|c}
\hline Modelos utilizados & Número & $\%$ \\
\hline Technology Acceptance Model - TAM & 06 & $16,20 \%$ \\
\hline Unified Theory of Acceptance and Use of Technology 2 & 03 & $8,10 \%$ \\
\hline Outros & 28 & $75,70 \%$ \\
\hline
\end{tabular}

Fonte: Autores.

Quanto às obras nos artigos analisados de acordo com a tabela 6, tiveram maior destaque a obra de Venkatesh (2003) com 09 citações, tratando da utilização de tecnologias; a obra de Davis (1989) com 07 citações, a qual trata do modelo teórico utilizado na maioria dos artigos analisados; a obra de Kotler (2002) com 05 citações, que trata da administração de marketing; também se destacou a obra de Yang (2013) sobre tecnologias móveis, tendo sido citada por três vezes nos artigos analisados e, por fim, a obra de Rogers (1962) sobre a difusão das inovações, também citada por 03 vezes nos artigos estudados.

Tabela 6 - Obras mais citadas.

\begin{tabular}{|c|c|c|}
\hline Autores & No. citação & Artigo \\
\hline Venkatesh ( 2003) & 09 & $\begin{array}{l}\text { VENKATESH, V.; MORRIS, M.G.; DAVIS, G.B.; DAVIS, F.D. User } \\
\text { acceptance of information technology: toward a unified view. MIS } \\
\text { Quarterly, v. } 27, \text { n. 3, p. 425-478, 2003. }\end{array}$ \\
\hline Davis (1989) & 07 & $\begin{array}{l}\text { DAVIS, F.D.; BAGOZZI, R.P.; WARSHAW, P.R. User acceptance of } \\
\text { computer technology: a comparison of two theoretical models. } \\
\text { Management Science, v. } 35 \text { n. 8, p. 982-1003, } 1989 .\end{array}$ \\
\hline Kotler (2002) & 05 & $\begin{array}{l}\text { KOTLER, P. Administração de Marketing. 12. ed. São Paulo: } \\
\text { Prentice-Hall do Brasil, } 2002 .\end{array}$ \\
\hline Yang (2013) & 03 & $\begin{array}{l}\text { YANG, Hongwei 'Chris'. Bon Appétit for apps: young American } \\
\text { consumers' acceptance of mobile applications. Journal of Computer } \\
\text { Information Systems, v. 53, n. 3, p. 85-96, } 2013 \text {. }\end{array}$ \\
\hline Rogers (1962) & 03 & ROGERS, E. M. Diffusion of Innovations. 1 ed. Glencoe: Free Press, 1962 \\
\hline
\end{tabular}

Fonte: Autores.

Na Tabela 7 com a análise, observou-se que os artigos mais apresentados nos eventos da EnANPAD, EMA e CLAV, referem-se a adoção de novas tecnologias em aplicativos móveis como smartphones e tablets nas mais diversas áreas. De 
acordo com os artigos analisados observou-se recentemente um significativo crescimento nos estudos no que tange à adoção de novas tecnologias no marketing. Estes estudos também trazem o comportamento do consumidor frente a esse novo modelo de mercado.

Para os autores ficou evidente a adoção de novas tecnologias como algo necessário devido à modernização das estruturas organizacionais, melhorando e eficiência dos serviços e produtos. Fica evidente também nos estudos a dificuldade da inserção de produtos tecnológicos e a aceitação do mesmo pelo consumidor. Em relação à inteligência artificial, de acordo com análise, teve um leve crescimento nas apresentações do evento da CLAV no útimo ano do estudo, parecendo ser um novo GAP do Marketing.

Tabela 7 - Tipos de novas tecnologias.

\begin{tabular}{l|c|c|c|c}
\hline Tipo de Inovação & Ema & Enampad & CLAV & Total \\
\hline $\begin{array}{l}\text { Aplicativos(smartphones, } \\
\text { tablets) }\end{array}$ & 03 & 01 & 15 & 18 \\
\hline Softwares & 01 & 05 & 08 & 14 \\
\hline Inteligencia artificial & 0 & 0 & 05 & 05 \\
\hline
\end{tabular}

Fonte: Autores.

\section{Considerações Finais}

Este estudo buscou analisar a adoção de novas tecnologias e os modelos utilizados para a introdução destes produtos tecnológicos no mercado. Este tema foi analisado a partir de um banco de dados publicados em eventos da EMA, EnANPAD e CLAV nos períodos de 2010 a 2018. Neste período foram apresentados 37 artigos que faziam referências ao tema de estudo.

Foi possível observar nestas publicações que a adoção de novas tecnologias se restringe a determinado tipo de produto ou serviço e também uma evolução rápida desta tecnologia no mercado. Observou-se que os modelos mais utilizados nos estudos analisados foram o TAM e UTAUT2, sendo que o primeiro trata basicamente da forma que o usuário aceita e usa a tecnologia, enquanto o segundo tenta explicar o comportamento do uso pelo usuário das tecnologias. Este fato demostra a importância na utilização de modelos para avaliar o consumo e a aceitação dos consumidores aos produtos ofertados.

Os resultados obtidos restringem-se apenas a 37 artigos apresentados em eventos como EMA e EnANPAD e a CLAV no período de 2010 a 2018 na qual o tema foi estudado. O tema é contemporâneo no Brasil, uma vez que o país teve uma adoção tecnológica tardia, diferenciando-se de países desenvolvidos que estão em outro patamar de desenvolvimento tecnológico. Podese, portanto, auferir que o estudo do tema no Brasil é recente, e apresenta limitações que prejudicam um estudo mais robusto.

Ao analisar os eventos supracitados observou-se um pequeno aumento na produção científica em um dos eventos (CLAV) entre o período de 2016 a 2018, apesar de existir uma lacuna observada no período de 2010 a 2015, pela ausência de informação disponível de artigos no sistema. Em relação à EnANPAD e a EMA pode-se constatar que no período de 2010 a 2018 as publicações foram inexpressivas em relação ao tema.

Os resultados dessa pesquisa demonstram que o tema objeto da pesquisa é emergente e que existe um grande campo de estudo para o desenvolvimento de estudos empíricos que tratem da adoção de novas tecnologias e Marketing. O Brasil por ser tardio em seu desenvolvimento tecnológico, havendo poucos estudos no que tange este tema torna difícil ter uma maior contribuição teórica, ou seja, os autores mais citados nos artigos se repetem frequentemente, tratando-se, portanto, dos mais renomados no assunto. 
Como limitação do presente estudo, destaca-se a utilização de apenas três bases de dados (EMA, EnANPAD E CLAV) pode-se sugerir novas pesquisas com uma maior amplitude, com abrangência em eventos, bem como em periódicos de outras bases de dados e até mesmo fazendo comparativos com publicações estrangeiras.

\section{Referências}

Araújo, C. F., Almeida, S. O. de. \& Bittencourt, R. (2018). Adoção de produtos tecnológicos por diferentes perfis de consumidores: uma análise por meio da escala technology a doption propensity. Rev. Bras. Gest. Neg. 21 (01). https://doi.org/10.7819/rbgn.v0i0.3955.

Bagozzi, R. P. (1975). Marketing as exchange. The Journal of Marketing, 32-39.

Bagozzi, R. P. \& Lee, K-H. (1999). Consumer resistance to, and acceptance of, innovations. Advances in Consumer Research, 26, $218-225$.

Bardin, L. (2009). Análise de conteúdo. Edições, 70.

Davis, F. D.; Bagozzi, R. P.; Warshaw, P. R. (1989). User acceptance of computer technology a comparison of two theoretical models. Management Science, 35(8).

Davis, M. M.; Aquilano, N. J. \& Chase, R. B. (2001). Fundamentos de administração da produção. (3a ed.), Bookman.

Ferreira, J. B., Rocha, A. da, \& Silva, J. F. (2014). Impacts of technology readiness on emotions and cognition in Brazil. Journal of Business Research, 67(5), $865-873$.

Gil, A. C. (2008). Métodos e técnicas de pesquisa social. (6a ed.), Editora Atlas S.A.

Gonçalves, C. A.; Filho, C. G. \& Neto, M. T. R. (2006). Estratégia empresarial: o desafio nas organizações. Saraiva.

Guo, B. et al. (2015). Mobile crowd sensing and computing: The review of an emerging human-powered sensing paradigm. ACM Computing Surveys (CSUR), 48(1), 7 .

HUNT, Sh. D. (1976). The nature and scope of marketing. Journal of Marketing, 40, 17- 28.

Kaefer, F.; Roper, J. \& Sinha, P. A. (2015). Software-assisted qualitative content analysis of news articles: example and reflections. Forum: Qualitative Social Research, 16

Kotler, Ph. \& Levy, S. J. (1969). Broadening the concept of marketing. The Journal of Marketing, 10-15.

Kotler, Ph. \& Levy, S. J. (1969). A new form of marketing myopia: rejoinder to Professor Luck. The Journal of Marketing, 55-57.

Loyola, S. (1999). A automação da fábrica: a transformação das relações de trabalho. Ed. do autor.

Luce, F. B. \& Souza, R. V. de. (2003). Atitudes dos Consumidores em Relação à Tecnologia: uma Análise Descritiva das Dimensões da Prontidão para Tecnologia.

Luck, D. J. (1969). Broadening the concept of marketing. Too far. The Journal of Marketing, 53- 55.

Macedo, M. A. S., Casanova, S. P., \& Almeida, K. (1999). Mapeamento e análise bibliométrica da utilização da análise envoltória de dados (DEA) em estudos das áreas de contabilidade e administração. In: Anais do $23^{\circ}$ ENANPAD. Foz do Iguaçu: ANPAD.

Masiero, G. (2007). Administração de empresas. Saraiva.

Mourad, A. I, Serralvo, F. A. \& João, B. do N. (2018). A marca como moderadora na aceitação de novas tecnologias: uma aplicação ao mobile banking, EnAMPAD.

Porter, M. E. (1986). Estratégia competitiva: técnicas para análise de indústrias e da concorrência. (7a ed.), Campus.

Richers, R. (1994). Recordando a infância do marketing brasileiro-um depoimento. Revista de Administração de Empresas, 34, $26-40$.

Rogers, E. M. (1976). New product adoption and diffusion. Journal of consumer Research.

Shaw, E. H. \& Jones, D. G. B. (2005). A history of schools of marketing thought. Marketing theory, 5, $239-281$.

Sheth, J. N. \& Gross, B. L. (1988). Parallel development of marketing and consumer behavior: a historical perspective. Historical perspectives in marketing, 9 33.

Venkatesh, V. et al. (2003). User acceptance of information technology: toward a unified view. MIS Quarterly, 27, 425-478.

Winner, L. (1994). Three paradoxes of the information age. Culture on the Brink. Ideologies of Technology, $191-197$.

Wohnrath, E. P. \& Gutierrez, V. C. P.(2007). O impacto da implantação de novas tecnologias de informação nas organizações, XIV Congresso Brasileiro de Custos - João Pessoa - PB, Brasil. 